\title{
A NOVEL LMNA MUTATION IN TWO SOUTH INDIAN SIBLINGS WITH HUTCHINSON-GILFORD PROGERIA SYNDROME
}

\author{
MUNSHI A. ${ }^{*}$, SATYANARAYANA U. ${ }^{2}$, BABU M.S. ${ }^{1}$, VASUNDHARA A. ${ }^{2}$, HARISCHANDRA Y.V. ${ }^{2}$, KRISHNA A. ${ }^{2}$, \\ KUMARI J.S. ${ }^{2}$ AND JYOTHY A. ${ }^{1}$ \\ ${ }^{1}$ Institute of Genetics and Hospital for Genetic Diseases, Osmania University, Begumpet, Hyderabad- 500016, AP, India. \\ 2Dr. Pinnamaneni Siddhartha Institute of Medical Sciences and Research Foundation, Chinnaoutpally- 521 286, AP, India. \\ *Corresponding Author: Email- anjanadurani@yahoo.co.in
}

Received: December 27, 2012; Accepted: January 03, 2013

\begin{abstract}
-
Background: Hutchinson-Gilford progeria syndrome is a rare genetic disorder characterized by dramatic premature senescence. Progeria is almost always caused by de novo point mutations in the LMNA gene that activates a cryptic splice donor site, producing a truncated mutant protein termed "progerin." A previous study has also reported an association of 594 C>T mutation in B4GALT1 gene with HGPS. In this study we investigated two HGPS siblings originating from South Indian region of Andhra Pradesh.
\end{abstract}

Methods: 50 healthy controls from the same ethnic group were also included in the study. Clinical characteristics of the affected children were noted down. All the exons of LMNA gene were amplified by PCR and the mutations detected by sequencing the PCR products. The 594 C>T mutation in B4GALT1 gene was also evaluated by sequencing the PCR products.

Results: A novel de novo point mutation C1699T was observed in exon 10 of LMNA gene in proband as well as the affected younger sibling. However, neither the parents nor the controls had this mutation.

Conclusions: The observation of heterozygous de novo LMNA mutation C1699T in HGPS patients in exon 10, supports the prevailing hypothesis that HGPS essentially represents a sporadic autosomal dominant disorder.

Keywords- Hutchinson-Gilford progeria syndrome, autosomal dominant disorder, LMNA gene, B4GALT1 gene

Citation: Munshi A., et al. (2013) A Novel LMNA Mutation in Two South Indian Siblings with Hutchinson-Gilford Progeria Syndrome. International Journal of Genetics, ISSN: 0975-2862 \& E-ISSN: 0975-9158, Volume 5, Issue 1, pp.-116-120.

Copyright: Copyright@2013 Munshi A., et al. This is an open-access article distributed under the terms of the Creative Commons Attribution License, which permits unrestricted use, distribution and reproduction in any medium, provided the original author and source are credited.

\section{Introduction}

Hutchinson-Gilford progeria syndrome (HGPS: MIM 176670), first described over a century ago, is an extremely rare genetic disorder, with features of accelerated and premature ageing [1-4]. The incidence of the disease has been reported to be about 1 in 4 to 8 million newborns with a total reported incidence of just over 100 in the century since its discovery [5]. Typically affected children appear normal at birth but begin to develop characteristic symptoms within the first year of life. The clinical features include alopecia, lypodystrophy, prominent joints, micrognathia, scleroderma like skin changes, prominent scalp veins, pluck bird appearance, beaked nose, short stature, often with premature death from myocardial infarction or stroke $[5,6]$. The age at death has been reported to range from 7-28 years with a median age of 13 years [7]. In some HGPS patients, the clinical presentation resembled mandibuloacral dysplasia with partial lypodystrophy which is caused by mutations in LMNA gene located on chromosome 1q21 encoding Lamin A/C $[8,9]$. Some HGPS patients had an inverted insertion of $1 \mathrm{q}$ while others had an interstitial deletion involving 1q23 [10]. Eriksson, et al. [2003] reported another HGPS patient with uniparental disomy of 1q involving the region flanked by markers D1S498 and D1S2836 harboring LMNA gene. Finally by means of homozygosity mapping and candidate gene analysis it became evident that heterozygous, recurrent de novo point mutations in the lamin A/C gene (LMNA: MIM 150330), caused this autosomal dominant disorder [11,12]. A few clinical reports and a LMNA mouse model also suggested the autosomal recessive inheritance of progeria $[4,5,13]$. Plasilova, et al. [2004] performed genome wide linkage analysis and subsequent LMNA mutation screening in a consanguineous family and provided molecular evidence for HGPS [5].

LMNA encodes two $A$ type lamins ( $A$ and $C$ ) which are the result of alternative splicing. They share the first 566 amino acids $[14,15]$. These two lamins along with $B$ type lamin represent the main components of the nuclear lamina. B type lamins are ubiquitiously expressed in all cell types and at all developmental stages. However, A-type lamins are absent in cells of early embryo, embryonic stem cells, cells of immune and hematopietic system as well as in the cells of neuroendocrine system $[5,16,17]$. In addition to HGPS, germline mutations in LMNA have been reported to cause 7 phenotypically different disorders, inherited in an autosomal dominant 
and/or recessive manner $[11,12,18]$. Brien and Weiss [2001] reported a novel $594 \mathrm{C}>\mathrm{T}$ silent mutation in $\beta(1-4)$ galactosyltransferase gene (B4GALT1) associated with HGPS in Caucasians [19]. The probands studied by them died of complications associated with coronary artery disease. Clark and Weiss [1995] reported that progeria types can be defined by differences in presentation of cell surface glycosylation that affects the binding of lectin DSA [20]. Studies support a model of defective synthesis of glycans with $\beta$ (14) N- acetylglycosamine linkages. Brein and Weiss [2001] reported that an allele of glycosyltransferase is linked to predisposition to HGPS [19].

However, the molecular mechanism by which these mutations produce the characteristic manifestations of this dominant disorder remains to be elucidated. In the present study we investigated two HGPS siblings originating from Andhra Pradesh region of South India. Clinical characteristics were evaluated and molecular analysis was carried out to detect mutations in LMNA as well as B4GALT1 genes.

\section{Materials and Methods \\ Subjects}

An 8 year old male child and his younger sibling, a 6 year old girl child presenting with HGPS were evaluated in the Dr. Pinnamaneni Siddhartha Institute of Medical Sciences and Research Foundation, Chinnaoutpally, Gannavaram, Krishna District, Vijayawada (A.P., India). The study was approved by the local ethical committee and the subjects were examined by a qualified pediatrician and clinical characteristics were noted down. Written informed consent was obtained from the family for molecular analysis. Routine biochemical investigations were carried out using standard procedures.

\section{DNA Isolation and Genotyping}

$5 \mathrm{ml}$ of peripheral blood was collected in EDTA tubes. The genomic DNA was extracted by standard phenol-chloroform method. All the exons of the LMNA gene were amplified by PCR using the primers listed in [Table-1] [21]. The PCR products were directly sequenced in forward and reverse direction using the Big Dye Terminator Cycle Sequencing Kit (Applied Biosystems) and analyzed on an automated sequencer (Applied Biosystems, Foster city, CA). The 594 C>T mutation in B4GALT1 gene was detected by amplifying the region of interest using following primers: Forward: 5' GCTCGTGGCAAAGCAGAACC 3'Reverse: 5'CCAGGCCTCACCTGGTTGATA-3' and subjecting the PCR products to direct sequencing as mentioned above. All the PCR products were also outsourced for sequencing to confirm the results.

Table 1- Primers for exons (1-11) of LMNA gene

\begin{tabular}{|lr|}
\hline Exon & Froward primer / Reverse primer \\
\hline 1 & M13F-CCGAGCAGTCTCTGTCCTTC / M13R-CCCTCTCACTCCCTTCCTG \\
2 & M13F-GCACTGTCTAGGCACACAGACT / M13R-GGGAGGGCCTAGGTAGAAGA \\
3 & M13F-TGTGACCCCTTTCCTCATC / M13R-CACTAGGGCAAGGGACTCAG \\
$4+5$ & M13F-GGCCTCCCAGGAACTAATTC / M13-RGTGGGGACACTTTTCATCCC \\
$6+7$ & M13F-CTTCCCCATACTTAGGGCCC / M13R-AAGGATGTTCCTCTCTCCAC \\
$8+9$ & M13F-GCAAGATACACCCAAGAGCC / M13R-GCTCCGATGTTGGCCATCAG \\
10 & M13F-GTAGACATGCTGTACAACCC / M13R-GGCCAGCGAGTAAAGTTCCA \\
11 & M13F-TTGGGCCTGAGTGGTCAGTC / M13R-GACCCGCCTGCAGGATTTGG \\
12 & M13F-ATCGAGGGGTAGGACGAGGT / M13R-TAAGGCAGATGTGGAGTTTCC \\
\hline
\end{tabular}

\section{Results}

An 8 year male child weighing $13 \mathrm{Kg}$, elder of the two siblings, born to second degree consanguious parents [Fig-1] was admitted for bronchopneumonia in Dr. Pinnamaneni Siddhartha Institute of Medical Sciences and Research Foundation in November 2011. The child was normal at birth and up to 1 year of age. Later he developed progressive skin, skeletal and other changes giving him characteristic HGPS appearance. The child had difficulty in walking since $5 \frac{1}{2}$ years and has been completely bed ridden since $71 / 2$ years of age.

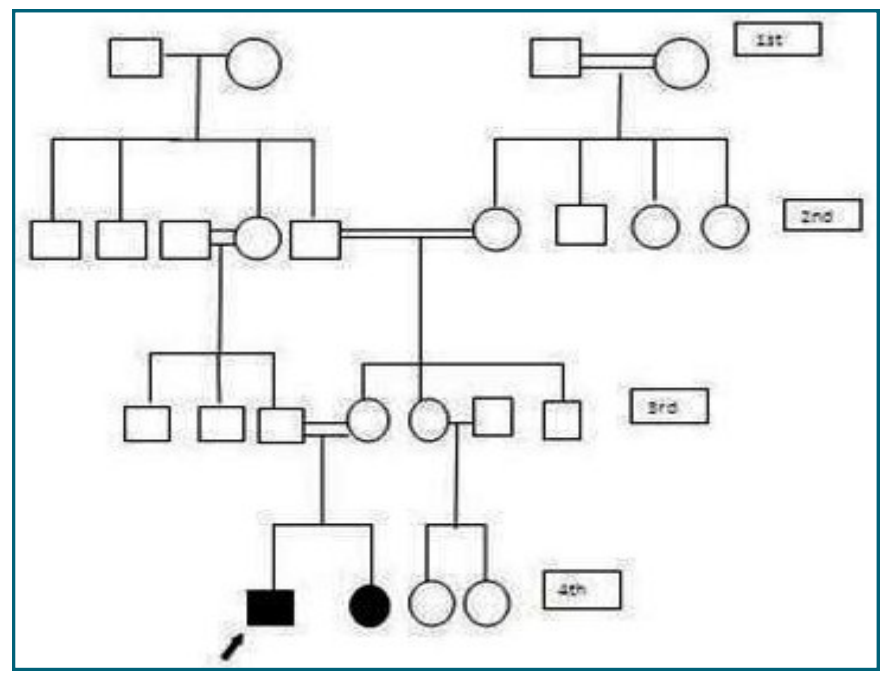

Fig. 1- Pedigree of the HGPS family

Child had difficulty for swallowing solids and was able to take only liquids and semisolids. He had fractures of both right humerus and right tibia with trivial trauma. All the clinical features of the affected child revealed age related features of HGPS [Fig-2a]. The head appeared to be large with a circumference of $49.5 \mathrm{~cm}$, had prominent scalp veins, alopecia started at 6th month of age. In addition to this, he had prominent eyes, receding mandible, narrow nasal bridge, crowding of teeth and pointed nasal tip, absent clavicles, pectus carinatum, flexion deformity of fingers, joint contractures, nail dystrophy, limited range of motion in joints of hands and legs, skin changes similar to scleroderma and loss of subcutaneous fat. $X$-ray photographs of the proband revealed $V$ shaped lytic lesions at the end of tibia, thinning of ribs and absent clavicles and severe osteopenia of the hand [Fig-2c].

Medical examination did not find any pathological findings in the parents. The mother of the proband did not suffer from any illness during pregnancy. However, the 6 year old younger girl sibling on physical examination revealed alopecia which started at 8th months of age. She had prominent scalp veins, characteristic facies with prominent eyes, sculpted nose and low set ears. The skin was dry and scaly with loss of subcutaneous fat, fixed flexion deformity of fingers and dystrophic nails. The child was walking without support but there was difficulty in sitting from standing posture. She had anterior fontanel patent (size $0.5 \times 0.5 \mathrm{~cm}$ ). All the clinical features of both the siblings have been summarized in [Table-2] [Fig-2a] [Fig2b].

\section{Biochemical Investigations}

Blood picture of the male child was normal $(\mathrm{Hb}: 12.8 \mathrm{gm} \%$; TLC: 30,200 per c.mm; platelet count: 3.3 lakhs per c.mm; normocytic, normochromic blood picture with neutrophilic leukocytes). The bilirubin level was $1.2 \mathrm{mg} / \mathrm{dl}$ and creatinine was $0.6 \mathrm{mg} / \mathrm{dl}$. The child had high total cholesterol $(208 \mathrm{mg} / \mathrm{dl})$, low HDL $(21 \mathrm{mg} / \mathrm{dl})$ and high LDL cholesterol (184 mg/dl). 


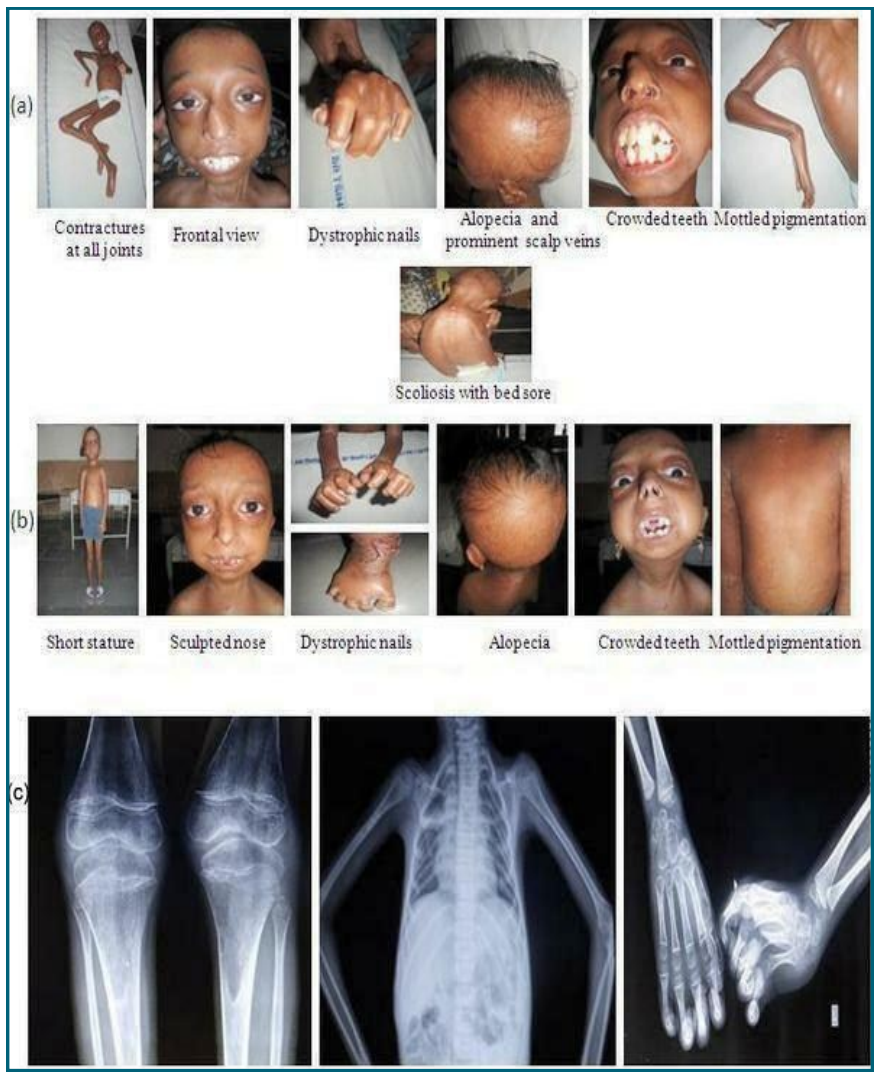

Fig. 2- Clinical features of the affected siblings: a) proband; b) younger sibling and c) X-ray photographs of the proband showing $\mathrm{V}$ shaped lytic lesions at the end of tibia, thinning of ribs \& absent clavicles and severe osteopenia of hand.

Table 2- Clinical features of the affected siblings

\begin{tabular}{|lcc|}
\hline Sex & Male & Female \\
\hline Age & 8 years & 6 years \\
Age of onset & After 1 year & 1.5 year of age \\
Height & $103 \mathrm{cms}<3$ rd percentile & $95.5 \mathrm{~cm}<3$ rd percentile \\
Weight & $13 \mathrm{kgs}<3$ rd percentile & $9 \mathrm{kgs}<3^{\text {rd }}$ percentile \\
BMl & $13<3^{\text {rd }}$ percentile & $9<3$ rd percentile \\
SMR & Prepubertal & Prepubertal \\
Eyebrows and eyelashes & Present & Present \\
Alopecia & Yes & Yes \\
Scleroderma & Yes & Yes \\
Prominent eyes & Yes & Yes \\
Pinched nose & Yes & Yes \\
Crowed teeth & Yes & Yes \\
Clavicles & Absent & Absent \\
Joint stiffness & Present & Present \\
Anterior fontanel & Closed & Present. Size $0.5 \times 0.5 \mathrm{~cm}$ \\
Dystrophic nails & Present & Present \\
Prominent scalp veins & Present & Present \\
Mottled pigmentation of the skin & Present & Present \\
Generalized lipodystrophy & Present & Present \\
\hline
\end{tabular}

\section{LMNA Mutation Analysis}

The mutational analysis was carried out for parents as well as affected children along with 50 healthy controls belonging to the same ethnic group. We sequenced all the exons of LMNA gene. The sequencing results revealed that both the siblings had a novel mutation C>T in exon 10 at $\mathrm{c} 1699$ (Gly567Trp) [Fig-3]. Both the siblings were heterozygous for the mutation. However, neither the parents nor the controls had this mutation revealing that this de novo point mutation is responsible for HGPS in these siblings. As far as the $594 \mathrm{C}>\mathrm{T}$ mutation in B4GALT1 is concerned, we did not find this mutation in the proband, his affected sibling as well the parents.

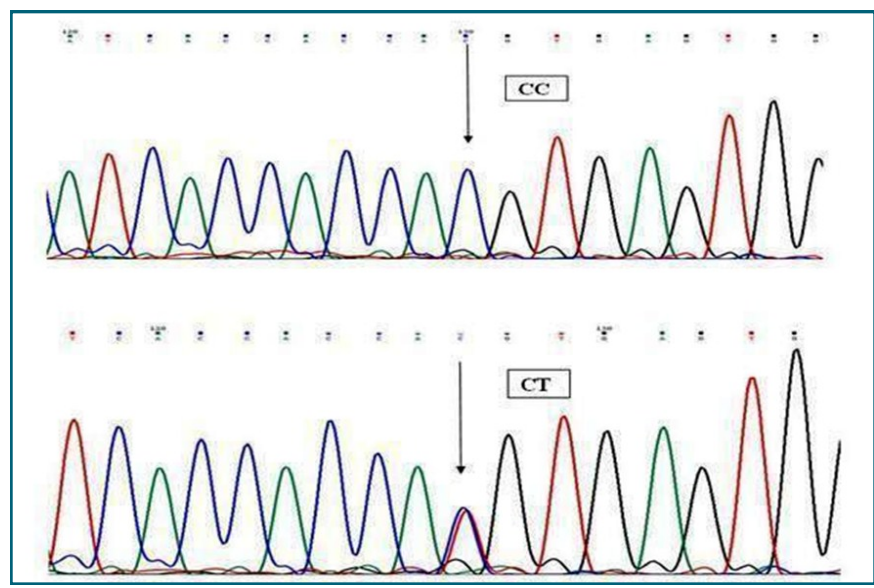

Fig. 3- Sequenced PCR products of the exon 10 of LMNA gene bearing the novel mutation showing $\mathrm{CC}$ homozygote in the parents and $\mathrm{CT}$ heterozygote in the affected siblings

\section{Discussion}

The cases described in the present study exemplified most of the characteristic features of HGPS. The blood picture of the proband was normal. However, he had high level of total cholesterol, high LDL and low HDL cholesterol suggestive of hyperlipidemia. Most of the children with HGPS have been reported to die from myocardial infarction and stroke, and hyperlipidemia is an established risk factor for these disorders.

A range of fascinating monogenic diseases including HGPS have been linked to mutations in the LMNA gene encoding A-type nuclear lamins, which are intermediate filamentous proteins of nuclear envelope. To date 29 sporadic HGPS cases with LMNA germline mutations have been reported $[11,12,22]$. Majority of these cases have been reported to carry heterozygous $\mathrm{G608G}$ splicing mutation. This was not detected in parents i.e. it occurred de novo. Unlike most other "accelerating aging diseases" which are caused by defective DNA repair, HGPS is an autosomal dominant disorder. In this study we present 2 HGPS siblings born to unaffected parents. The children were born to parents with second degree consanguinity. Consanguinity has been reported twice in classical HGPS $[23,24]$. Plasilova, et al. [2004] have provided molecular evidence that HGPS is also inherited in autosomal recessive mode of inheritance in consanguious families with more than one affected sibling [5]. Although recurrence in classical HGPS (autosomal dominant disorder) has not been described, D'Apice, et al. [2004] found a parental origin of the mutations in LMNA gene [25]. In a classical HGPS patient with G608G mutation, the phenotypically normal mother was found to have somatic mosaicism. $10 \%$ of her buccal cells were reported to harbor the same mutation [26]. A segregation study confirmed that the mutation was transmitted from mother, who showed germ line as well as somatic mosaicism. In the present study we found recurrence of the disease which might be as a result of germ line mosaicism in one of the parents. This is for the first time that we found recurrence of the disease in case of HGPS inherited in an autosomal dominant fashion. The drawback of the study is that we were not able to confirm somatic or germ line mosaicism in the parents. 
Chromosomal abnormalities have also been reported in few HGPS cases. However, in the present study, the parents as well as the affected children had a normal karyotype. We sequenced all the exons of LMNA gene, but did not find any mutation in exons11, 9, 8 and 2 as reported previously [Table-3]. Both the siblings with characteristic HGPS appearance had a novel C1699T mutation in exon 10 of LMNA gene. The results were confirmed by outsourcing the samples for DNA sequencing. In addition to G608G (C1824T) mutation, Eriksson, et al. [2003] reported another case with G608S (1822 G>A) mutation which was simultaneously reported by two other groups $[12,27]$. These mutations have been reported to activate a cryptic splice donor site resulting in the synthesis of a protein with 50 amino acids deleted near the carboxyl terminus of prelamin A. This truncated variant is not appropriately processed to lamin A. During processing of the mutated prelamin-A, a protein metabolite called progerin gets accumulated. This metabolite induced nuclear blebbing in HGPS cells grown in culture [16]. This suggests serious disruption of DNA metabolism may be occurring in these cells and several outcomes like induction of cell senescence, induction of apoptosis, dysregulation of gene expression, dysregulation of differentiation etc. are possible. Progerin has also been reported to interfere with mitosis and induces DNA damage responses [27,28]. The novel mutation in the present study might give rise to a cryptic splice variant of LMNA protein. This novel mutation was not observed in parents as well as the healthy controls.

Table 3- Mutations reported in LMNA gene in HGPS

\begin{tabular}{|lcc|}
\hline Exon & Mutation & Reference \\
\hline 2 & G433A & 11 \\
8 & C1623 T & 27 \\
9 & C1791 T & 27 \\
10 & G1626C & 5 \\
& C1699T & This study \\
11 & C1824 T & $5,11,27$ \\
& G1822A & 5,27 \\
& C1868 G & 7 \\
\hline
\end{tabular}

Patients with mutations in exon 10 and 11 have typical phenotype of HGPS, while as mutations in exon 2, 8 or 9 (compound heterozygote) show unusual clinical features like ample subcutaneous tissues and persistence of coarse hair on the head (for exon 2 mutation) and absence of coronary artery disease (for exon 8 and 9 mutations). However, the only report on mutations in exon 10 of LMNA gene has not mentioned anything about the presence or absence of coronary artery disease. In the present case also we did not find any evidence of coronary artery disease in the affected children [5]. However, the proband had many episodes of bronchopneumonia. All the LMNA mutations reported so far have been summarized in table 3. A two year Dutch boy with HGPS was a compound heterozygote for two mutations in LMNA gene [29].

B4GALT1 gene is one of the seven beta 1-4-galactosyltransferase (beta4Galt) genes. These genes encode for type-II membrane based glycoproteins. These proteins appear to have exclusive specificity for the donor substrate UDP-galactose. B4GALT1 gene is unique among these genes because it encodes for an enzyme that participates both in glycoconjugate and lactose biosynthesis. $A$ novel mutation $594 \mathrm{C}>\mathrm{T}$ has been reported to be associated with HGPS [19]. However, in the present study we did not find this mutation in any of the subjects.

At present there is no particular treatment for progeria. However, more research is warranted to find effective treatments and/or a cure for children affected with HGPS. The results of the present study suggest that de novo point mutations in LMNA gene are responsible for causing HGPS. This supports the prevailing hypothesis that HGPS represents the sporadic autosomal dominant disorder.

\section{Acknowledgements}

We are thankful to Siddhartha Academy of General and Technical Education Vijayawada for providing hospital facilities. We are also grateful to Dr. C. Nageswara Rao and Dr. Sudha. D. Deshpande for their constant support and encouragement. The financial assistance from DBT, New Delhi is acknowledged with thanks.

\section{References}

[1] Hutchinson J. (1886) Lancet, I, 923.

[2] Gilford H. (1904) Practitioner, 73, 188-217.

[3] McKusick V.A. (1952) The American Journal of Syphilis, 36, 101-126.

[4] DeBusk F.L. (1972) Journal of Pediatrics, 80, 697-724.

[5] Plasilova M., Chattopadhyay C., Pal P., Schaub N.A., Buechner S.A., Mueller H. Miny P, Ghosh A, Heinimann K. (2004) Journal of Medical Genetics, 41, 609-614.

[6] Merideth M.A., Gordon L.B., Clauss S., Sachdev V., Smith A.C.M., Perry M.B., et al. (2008) New England Journal of Medicine 358, 592-604.

[7] Fukuchi K., Katsuya T., Sugimato K., Kuremura M., Kim H.D., Li L., et al. (2004) Journal of Medical Genetics, 41(5), e67.

[8] Parkash H., Sidhu S.S., Raghavan R., Deshmukh R.N. (1990) American Journal of Medical Genetics, 36, 431-433.

[9] Novelli G., Muchir A., Sangiuolo F., Helbing-Lecrelerc A., D'Apice M.R., Massart C., et al. (2002) American Journal of Human Genetics, 71, 426-431.

[10]Luengo W.D., Martinez A.R., Lopez R.O., Basalo C.M., RojasAtencio A., Quintero M., et al. (2002) American Journal of Medical Genetics, 113, 298-301.

[11]Eriksson M., Brown W.T., Gordon L.B., Glynn M.W., Singer J., Scott L., et al. (2003) Nature, 423, 293-298.

[12]De Sandre-Giovannoli A., Bernard R., Cau P., Navarro C., Amiel J., Boccaccio I., et al. (2003) Science, 300, 2055.

[13]Maciel A.T. (1988) American Journal of Medical Genetics, 31, 483-487.

[14]Fisher D.Z., Chaudhary N., Blobel G. (1986) Proceedings of the National Academy of Sciences, 83, 6450-6454.

[15]McKeon F.D., Kirschner M.W., Caput D. (1986) Nature, 319,463 $-468$.

[16]Goldman R.D., Shumaker D.K., Erdos M.R., Eriksson M., Goldman A.E., Gordon L.B., et al. (2004) Proceedings of the National Academy of Sciences, 101, 8963-8968

[17]Mounkes L.C., Kozlov S., Hernandez L., Sullivan T., Stewart C.L. (2003) Nature, 423, 298-301.

[18]Burke B., Stewart C.L. (2002) Nature Reviews Molecular Cell Biology, 3, 575-585.

[19]O'Brien M.E. and Weiss M.E. (2001) Human Mutation, 17(4), 231-234.

[20]Clark M.A. and Weiss A.S. (1995) Biochimica et Biophys Acta., 
$120,142-148$.

[21]De Sandre-Giovannoli A., Chaouch M., Kozlov S., Vallat J.M., Tazir M., Kassouri N. et al.(2002) American Journal of Human Genetics, 70(3), 726-736.

[22]Cao K., Graziotto J.J., Blair C.D., Mazzulli J.R., Erdos M.R., Krainc D., et al. (2011) Science Translational Medicine, 3, 89 ra58.

[23]Bhakoo O.N., Garg S.K., Sehgal B. (1964) Indian Pediatrics, 2, 164-169.

[24]Broc R., Nicolle M., De Beaujeu J. (1935) Journal Presse. Med., 39, 786-788.

[25]D'Apice M.R., Tenconi R., Mammi I., van den Ende J., Novelli G. (2004) Clinical Genetics, 65, 52-54.

[26]Wuyts W., Biervliet M., Reyniers E., D'Apice M.R., Novelli G., Storm K. (2005) American Journal of Medical Genetics, 135A, 66-68.

[27]Cao H., Hegele R.A. (2003) Journal of Human Genetics, 48, 271-274.

[28]Liu Y., Rusinol A., Sinensky M., Wang Y., Zou Y.A. (2006) Journal Cell Science, 19, 4644-4649.

[29]Verstraeten V.L.R.M., Broers J.L.V., van Steensel M.A.M., ZinnJustin S., Ramaekers F.C.S., Steijlen P.M., et al. (2006) Human Molecular Genetics, 15, 2509-2522. 